\title{
Cumplimiento de las recomendaciones de práctica de actividad física en función de los cutoffs points y el género en estudiantes universitarios españoles
}

\author{
Compliance of physical activity guidelines depending on \\ cutoffs points and gender in Spanish university students
}

\author{
Cristina Corella*, Sheila Rodríguez-Muńoz, Alberto Abarca-Sos y Javier Zaragoza
}

Universidad de Zaragoza. Expresión musical, plástica y corporal

\begin{abstract}
Resumen. Los principales objetivos de este estudio fueron: a) estudiar los niveles de actividad física (AF) según los diferentes puntos de corte utilizados y el género; b) analizar el cumplimiento de las diferentes recomendaciones internacionales de AF según el género y puntos de corte. 95 universitarios, 33 hombres y 62 mujeres $(21,96$ años $\pm 2,33)$ llevaron acelerómetro para medir sus niveles de AF utilizando puntos de corte uniaxiales y triaxiales. Los resultados seńalan mayores niveles de AF en los hombres para la AF vigorosa, pero no en la moderada. La mayoría de la muestra cumple con las recomendaciones de AF moderada, sin embargo, en la AF vigorosa el cumplimiento es bajo. El análisis de la AF con puntos de corte triaxiales son mayores a los uniaxiales, así como un mayor porcentaje de cumplimiento cuando se utilizan las recomendaciones establecidas por la Physical Activity Guidelines Advisory y la World Health Organization.

Palabras clave: recomendaciones, estudiantes universitarios, actividad física, acelerómetros, puntos de corte.
\end{abstract}

Abstract. The main objectives of this study were: a) study the levels of physical activity (PA), according to the different cutoffs points used and gender; b) analyze the compliance of different international recommendations for physical activity by gender and cutoff points. 95 university students, 33 men and 62 women (21, 96 years) wore accelerometers to assess their levels of PA using uniaxial and triaxial cutoffs points. Results indicated higher PA levels in men in vigorous intensity, not in moderate. A high percentage of the sample fulfills the recommendations for moderate PA, nevertheless, for vigorous PA, it is low. Analyzes of PA with triaxial cutoffs points are higher than the uniaxial ones, besides a greatest compliance of PA recommendations of Physical Activity Guidelines Advisory (2008) and World Health Organization (2010) recommendations are used.

Keywords: recommendations, college students, physical activity, accelerometers, cut points

\section{Introducción}

La literatura científica constata que en la última fase de la adolescencia y en la primera fase de la etapa adulta se produce un descenso en los niveles de actividad física (NAF) (López Sánchez, González Víllora, Díaz Suárez, 2016; López Sánchez, Ahmed, Díaz Suárez, 2017;Cocca, Liukkonen, Mayorga y Viciana, 2014; Ortega, et al., 2013), siendo el paso a la universidad una etapa clave en el desarrollo de una buena calidad de vida (Timperio, Salmon \& Ball, 2004). A nivel internacional, los datos sobre los NAF en población universitaria, señalan que más de la mitad de los estudiantes no realiza la suficiente actividad física (AF), sobre todo en el género femenino, para que genere beneficios en su salud, independientemente del país de origen (Irwin, 2004). Concretamente, entre el 30\%$50 \%$ de los estudiantes universitarios estudiados no satisfacen el cumplimiento de las recomendaciones establecidas (Racette, Deusinger, Strube, Highstein \& Deusinger, 2005). Ejemplo de ello es el trabajo realizado en diferentes países europeos por Hasse, Steptoe, Phil, Sallis y Wardle (2004), donde constataron que entre el $23-43 \%$ de los estudiantes universitarios, eran inactivos en su tiempo libre.

Dirección para correspondencia [Correspodence address]: Cristina Corella. E-mail: criscor@unizar.es
Los datos en población universitaria española están en la misma sintonía. En el estudio realizado entre universitarios por Romaguera, Tauler, Bennasar, Pericas, Moreno, Martínez y Aguilo (2011), se constata que solo el $43 \%$ de los sujetos, mostraba niveles adecuados de AF, encontrando diferencias según el género ( $55 \%$ en el caso de los hombres frente al 34\% en las mujeres). Mientras, Varela-Mato, Cancela, Ayan, Martín, y Molina (2012), encontraron que solamente el 27,4\% de la población universitaria estudiada se podía considerar activa. En otro estudio realizado por Práxedes, Sevil, Moreno, del Villar y García-González (2016), concluyeron que el $51,39 \%$ de los estudiantes universitarios estudiados, no alcanzaban los 30 minutos diarios de práctica de actividad física moderada-vigorosa (AFMV) recomendados, siendo el grado de cumplimiento superior en los hombres.

A tenor de los diferentes resultados podemos afirmar que la etapa universitaria es un período crítico, por un lado por el descenso en la práctica de AF, pero por otro, hay que considerar la posible relación entre el estilo de vida de la etapa universitaria y los comportamientos que se van manifestando tanto a lo largo de la etapa joven como adulta (Hultquist, Duckham, Stinson \& Thompson, 2009; Molina-García, Castillo $\&$ Pablos, 2009). Además, algunos estudios sugieren que la 
AF realizada durante el último año universitario, puede ser uno de los predictores más influyentes de la AF en la etapa post-universitaria (Sparling \& Snow, 2002).

En los últimos ańos han sido publicadas diferentes recomendaciones de práctica de AF para población adulta. The Centers for Disease Control and Prevention (CDC) y el American College of Sports Medicine (ACSM) (Pate, et al., 1995), recomendaron que cada adulto debería acumular 30 minutos o más de AF de moderada intensidad durante todos los días de la semana. La American Heart Association (AHA) and ACSM (Haskell, et al., 2007), especificaron que la AF aeróbica a intensidad moderada, debería realizarse durante un mínimo de 30 minutos, cinco días a la semana y la actividad física vigorosa (AFV), durante un mínimo de 20 minutos, durante tres días a la semana, especificando la posibilidad de combinar la AFMV. Así mismo, añaden que el trabajo de fortalecimiento muscular debe realizarse semanalmente en dos días no consecutivos, de 8-10 ejercicios y de 8-12 repeticiones. En el año 2008, la Physical Activity Guidelines Advisory (PAG) (2008), recomendó realizar una práctica de AF durante un mínimo de 150 minutos semanales a una intensidad moderada (en fracciones de 10 min mínimo), o 75 minutos a la semana a una intensidad vigorosa. Los ejercicios de fortalecimiento muscular deberían involucrar a todos los grandes grupos musculares y realizarse dos días a la semana (Oja, Bull, Fogelholm \& Martin, 2010). Posteriormente la World Health Organization (WHO) en 2010, establece las mismas recomendaciones para adultos que la PAG (2008), e invita a que los 150 minutos semanales de actividad física moderada (AFM), se distribuyan a lo largo de toda la semana, lo que favorecerá la integración de la práctica de AF en la vida cotidiana.

Atendiendo a los estudios que han utilizado estas recomendaciones, podemos afirmar que muy pocos adultos, incluida la población universitaria, cumplen, independientemente del organismo que las proponga, y además, es menos probable que lo hagan conforme aumenta la edad (e.g., Cocca, Mayorga \& Viciana, 2013; Colley, Garriguet, Janssen, Craig, Clarke, et al., 2011; Romaguera, et al., 2011; Troinano, Berrigan, Dodd, Mâsse, Tilert, et al., 2008).

Para conocer los NAF y analizar el grado de cumplimiento de las recomendaciones de $\mathrm{AF}$, se han utilizado diferentes métodos de medición, aunque predomina la utilización de métodos subjetivos lo que afectará la validez de los resultados finales (Prince, Adamo, Hamel, Hardt, Connor, et al., 2008), ya que la medición subjetiva puede subestimar o sobreestimar los NAF (Downs, Van Hoomissen, Lafrenz \& Julka, 2014). El acelerómetro se ha utilizado en numerosos estudios con diversa población, mostrándose válido y fiable para la población adulta joven (Matthews, 2005). Sin embargo, pocos estudios han utilizado esta medida objetiva para medir los NAF en población universitaria española (Arias-Palencia, Solera-
Martínez, Gracia-Marco, Silva, Martínez-Vizcaíno, CañeteGarcía-Prieto \& Sánchez-López, 2015).

Pero no solo los NAF pueden verse condicionados por el instrumento utilizado para medirla, sino que también puede hacerlo, cuando utilizando una medida objetiva como es el acelerómetro, se utilicen diferentes epoch para programar el acelerómetro (Yıldırım, et al., 2011), o diferentes puntos de corte (cutoffs points), entendiendo los puntos de corte como la unidad que nos permite clasificar las diferentes intensidades de la AF. Existen diferentes puntos de corte, unos para datos uniaxiales (Freedson, Melason \& Sirard, 1998) y otros para datos triaxiales (Sasaki, John \& Freedson, 2011), según los ejes utilizados para medir la AF por parte de los acelerómetros. La utilización de unos u otros, puede condicionar sustancialmente los resultados del estudio, ya que según encontraron Watson, Carlson, Carrol y Fulton (2014), había una variación en el porcentaje de sujetos que cumplían las recomendaciones de una muestra de adultos de hasta un 90\% en función de los puntos de corte que se utilizaban para analizar los datos de los acelerómetros.

Es por ello, que los objetivos planteados en este estudio son: a) Estudiar las diferencias según género y puntos de corte utilizados, en los niveles de actividad física moderadavigorosa medidos mediante acelerómetros, de una muestra de estudiantes universitarios espańoles; b) analizar el grado de cumplimiento de las diferentes recomendaciones internacionales de actividad física según el género.

Las principales hipótesis son: 1) Los niveles de actividad física moderada y/o vigorosa (medida en minutos/semana), variará según los puntos de corte utilizados (uniaxiales y triaxiales) y según el género, siendo mayor en hombres que en mujeres; 2) El porcentaje de participantes que cumple las recomendaciones de prácticas de AF será bajo, variando según la recomendación de práctica utilizada, encontrando diferencias significativas a favor del género masculino.

\section{Método}

\section{Participantes}

Se ofertó la participación en el estudio a todos los estudiantes de tercero y cuarto curso de los diferentes grados impartidos en el campus de Teruel, perteneciente a la Universidad de Zaragoza: Ingeniería electrónica y automática, Ingeniería informática, Bellas artes, Psicología, Administración y dirección de empresas, Educación Primaria e Infantil. La muestra inicial se compuso de 145 estudiantes universitarios (53 hombres, 92 mujeres). Esta muestra inicial se redujo al aplicar los siguientes criterios de inclusión: rotura de acelerómetros (ocho participantes excluidos); monitorización durante al menos un día del fin de semana (seis participantes fueron excluidos); monitorización de al menos cuatro días entre se- 
mana (seis participantes excluidos); monitorización de al menos 600 minutos cada día (30 participantes excluidos). No existe un consenso en la literatura en relación al tiempo diario que un sujeto tiene que llevar puesto el acelerómetro, ya que diferentes autores consideran que seis horas es suficiente y otros 10 horas, así como diferentes criterios para un día entre semana, 10 horas, o un día de fin de semana, ocho horas (Trost, McIver \& Pate, 2005). En el estudio se ha tenido en consideración el criterio más exigente, en el que es necesario que se haya registrado por lo menos 10 horas. De igual modo, no hay un consenso en el mínimo número de días de registro necesario para que el sujeto sea considerado válido, pudiendo variar entre tres y siete días (Ridgers \& Fairgclough, 2011). En este estudio se ha considerado que tendrían que ser cinco días mínimo, con al menos cuatro días de registro válidos entre semana y un día del fin de semana. A partir de la aplicación de estos criterios, la muestra final fue de 95 estudiantes universitarios (33 hombres y 62 mujeres) con una media de edad de $21,96 \pm 2,33$ años.

\section{Instrumentos}

Acelerómetros. La AF fue medida durante 7 días consecutivos (de lunes a domingo) utilizando los acelerómetros Actigraph GT3X y GT3X+. Un mínimo de cuatro días entre semana (mínimo de $600 \mathrm{~min} /$ día registrados) y de un día del fin de semana fueron utilizados como criterios de inclusión (Trost, et al., 2005). Para el presente estudio se han utilizado un epoch de 10 segundos, que posteriormente se han agrupado en un minuto, considerado como un intervalo válido para la población adulta (Matthews, Hagstromer, Pober \& Bowles, 2012; Trost, et al., 2005). Para descargar los datos registrados por los acelerómetros se utilizó el software Actilife 6.1. (Actrigaph, Pensacola, FL, USA). Los counts registrados se transformaron a minutos/día de actividad usando dos puntos de corte diferentes, uno para datos uniaxiales y otro para datos triaxiales. Para los datos registrados mediante acelerómetros uniaxiales, se utilizaron los puntos de corte de Freedson et al., (1998): actividad sedentaria 0-99, actividad ligera $\leq 1951$, moderada 1952-5724, vigorosa 5725-9498 y muy vigorosa $\geq 9499$ counts $\mathrm{min}^{-1}$, previamente utilizados en otros estudios (Craig, et al., 2003; Viñas, Barba, Ngo \& Majem, 2013). Para los datos recogidos mediante acelerómetros triaxiales, se han seleccionado los puntos de corte de Sasaki et al., (2011): actividad ligera 0-2690, moderada 2690-6166, vigorosa 6167-9642 y muy vigorosa $>9642$ counts $\mathrm{min}^{-1}$, utilizados también en otros estudios previos (Fritschi, Brown \& Van Uffelen, 2014).

Para conocer el grado del cumplimiento de las recomendaciones, sumamos los minutos de AFMV semanales. Las diferentes recomendaciones utilizadas fueron:

Recomendación uno (R1): AFM un mínimo de 30 minu- tos, cinco días a la semana; AFV un mínimo de 20 minutos, tres días a la semana (ACSM; AHA, 2007).

Recomendación dos (R2): AFM un mínimo de $150 \mathrm{mi}-$ nutos semanales; AFV un mínimo de 75 minutos semanales (PAG, 2008; WHO, 2010).

\section{Procedimientos}

Este estudio se realizó durante los meses de Septiembre a Noviembre de 2014, con la aprobación del Comité Ético de Investigación de Aragón (CEICA).

Los participantes fueron informados de los objetivos del estudio. Aquellos que quisieron participar firmaron previamente el consentimiento.

Se informó a cada participante que llevará el acelerómetro en la cadera derecha (Trost,et al.,2005; Matthews, et al., 2012) desde la primera hora de la mañana hasta antes de acostarse durante un total de siete días consecutivos, quitándoselo únicamente para dormir y durante las actividades acuáticas (Welk, 2002). Los estudiantes que decidieron participar, recibieron una hoja informativa sobre el uso de los acelerómetros y una ficha para poder registrar las incidencias que pudieran acontecer durante los siete días de monitorización.

\section{Análisis estadístico de los datos}

Hemos realizado análisis descriptivos en función del género, de los NAF (minutos semanales en AFMV) y de las recomendaciones de práctica de AF según los distintos puntos de corte para datos uniaxiales y triaxiales. Se realizaron las pruebas de normalidad de Kolmogorov Smirnov, con el resultado de normalidad de la muestra. Realizamos un ANOVA para contrastar las diferencias por género en NAF y una prueba $T$ para muestras relacionadas para analizar las diferencias entre los puntos de corte uniaxiales y triaxiales según las recomendaciones seleccionadas. Además efectuamos un análisis chi cuadrado, para ver si existen diferencias según el género, respecto al grado de cumplimiento de las recomendaciones. Todos los análisis de datos fueron realizados con el programa informático SPSS versión 21.0. El nivel alfa se fijó en $p<.05$ para todos los análisis.

\section{Resultados}

En la tabla 1, se muestra la media de minutos y la desviación típica de los minutos semanales de práctica de AFM y AFV llevada a cabo por los sujetos participantes según los puntos de corte utilizados. La AFM realizada por la muestra estudiada está muy por encima de los $150 \mathrm{~min} / \mathrm{semanales} \mathrm{recomen-}$ dados en ambos géneros, independientemente de los puntos de corte utilizados. Mientras, la AFV solo está por encima de los $60 \mathrm{~min} / \mathrm{semanales} \mathrm{recomendados} \mathrm{en} \mathrm{la} \mathrm{R1} \mathrm{y} \mathrm{de} \mathrm{los} 75$ 
min/semanales de la R2, en el caso de los hombres y cuando utilizamos los puntos de corte para datos triaxiales. Encontramos diferencias significativas $(p<, 01)$ solo en la AFV entre chicos y chicas, a favor de los chicos, independientemente de los puntos de corte utilizados.

Cabe destacar que las medias de minutos semanales de AFM y AFV, siempre son mayores cuando se utilizan los puntos de corte para datos triaxiales, tanto en hombres como en mujeres. Tal y como se muestra en la tabla 2 , encontramos diferencias estadísticamente significativas $(p<, 001)$ en la AFM y también en la AFV entre los puntos de corte para datos uniaxiales o trixiales.

En la tabla 3 se muestra el número y porcentaje de sujetos que cumplen las distintas recomendaciones de práctica de AF y en función de los puntos de corte utilizados. Considerando los puntos de corte para datos uniaxiales y en relación a la AFM, la R1 fue alcanzada por el $67 \%$ del total de universitarios, mientras que en la R2, el porcentaje se sitúa en el $94 \%$. Si utilizamos los puntos de corte para datos triaxiales, el porcentaje de sujetos que cumplen la R1 es del 90\% mientras que el porcentaje, según la R2 es del 98\%. Si hablamos de la AFV, la R1 fue alcanzada por el $4 \%$ (según puntos de corte para datos uniaxiales) y por el $12 \%$ si tenemos en cuenta la R2. Si utilizamos los puntos de corte para datos triaxiales, el porcentaje de sujetos que cumplen la R1 es del 14\% frente al 27\% de la R2. Cabe destacar que los porcentajes de cumplimiento de las recomendaciones, son siempre mayores cuando se utilizan los puntos de corte para datos triaxiales en ambos géneros.

Solamente el análisis de los datos referentes a la AFV, muestra diferencias significativas en la AFV entre chicos y chicas, a favor de los chicos, tanto si utilizamos los puntos de corte para datos uniaxiales como para los datos triaxiales y con las diferentes recomendaciones de AF. La significatividad es mayor cuando se toma como referencia la $\mathrm{R} 2\left[\chi^{2}=14,30\right.$, $p<, 001$ puntos de corte para datos uniaxiales y $\chi^{2}=23,21, p$ $<.001$ utilizando puntos de corte para datos triaxiales], respecto a la $\mathrm{R} 1\left[\chi^{2}=7,84, p<, 05\right.$ puntos de corte para datos uniaxiales y $\chi^{2}=9,75, p<, 05$ cuando utilizamos puntos de corte para datos triaxiales]. Es reseñable que en ambos géneros pero sobre todo en el caso de las mujeres, el bajo porcentaje de cumplimiento de las recomendaciones respecto a la AFV (en el caso de las mujeres oscila entre el $0 \%$ y el $11 \%$ ), siendo menor todavía si utilizamos los puntos de corte para datos uniaxiales.

Tabla 1. Descriptivos (media y desviación estándar) y diferencias según de los Niveles de Actividad Física (min) en función de los diferentes puntos de corte e intensidades.

\begin{tabular}{lcccccccc}
\hline & \multicolumn{2}{c}{$\begin{array}{c}\text { AFM semanal } \\
\text { Uniaxiales }\end{array}$} & \multicolumn{2}{c}{$\begin{array}{c}\text { AFM semanal } \\
\text { Triaxiales }\end{array}$} & \multicolumn{2}{c}{$\begin{array}{c}\text { AFV semanal } \\
\text { Uniaxiales }\end{array}$} & \multicolumn{2}{c}{$\begin{array}{c}\text { AFV semanal } \\
\text { Triaxiales }\end{array}$} \\
\cline { 2 - 10 } & $M$ & $D T$ & $M$ & $D T$ & $M$ & $D T$ & $M$ & $D T$ \\
\hline Hombres & 378,06 & 176,02 & 483,43 & 177,91 & $53,55^{* *}$ & 44,67 & $96,72^{* *}$ & 60,26 \\
Mujeres & 325,78 & 118,03 & 463,60 & 144,83 & 19,88 & 28,89 & 42,71 & 41,26 \\
Total & 343,94 & 142,18 & 470,49 & 156,45 & 31,58 & 38,48 & 61,47 & 54,86 \\
\hline
\end{tabular}

${ }^{* *} p<0,01 * p<0,05$; los asteriscos hacen referencia a las diferencias por género.

Tabla 2. Diferencias entre los puntos de corte uniaxiales y triaxiales en cada una de las intensidades.

\begin{tabular}{|c|c|c|c|c|c|c|c|c|}
\hline \multirow[b]{3}{*}{ Diferencias entre puntos de corte } & \multicolumn{4}{|c|}{$\begin{array}{c}\text { Diferencias entre AFM en función de los puntos } \\
\text { de corte (uniaxiales y triaxiales) }\end{array}$} & \multicolumn{4}{|c|}{$\begin{array}{c}\text { Diferencias entre AFV en función de los puntos } \\
\text { de corte (uniaxiales y triaxiales) }\end{array}$} \\
\hline & $\mathrm{T}$ & $\mathrm{gl}$ & $\begin{array}{c}\text { Intervalos de } \\
\text { confianza }\end{array}$ & P (Sig. Bilateral) & $\mathrm{T}$ & $\mathrm{gl}$ & $\begin{array}{c}\text { Intervalos de } \\
\text { confianza }\end{array}$ & P (Sig. Bilateral) \\
\hline & 17,96 & 94 & $112,56 \quad 140,54$ &, 000 & 11,67 & 94 & $24,67 \quad 35,10$ &, 000 \\
\hline
\end{tabular}


Tabla 3. Grado de cumplimiento en función de las diferentes recomendaciones de práctica de AF, de los puntos de corte uniaxiales y triaxiales, del género y de las diferentes intensidades de práctica.

\begin{tabular}{|c|c|c|c|c|c|c|c|c|c|c|c|c|c|c|c|c|}
\hline & \multicolumn{4}{|c|}{$\begin{array}{c}\text { R1: ACSM (2007) } \\
\text { AFM: } 30 \text { min } 5 \text { días a la } \\
\text { semana }^{\mathrm{R} 1}\end{array}$} & \multicolumn{4}{|c|}{$\begin{array}{c}\text { R2: PAG (2008) y WHO } \\
(2010) \\
\text { AFM: } 150 \text { min semanales }{ }^{\mathrm{R} 2}\end{array}$} & \multicolumn{4}{|c|}{$\begin{array}{c}\text { R1: ACSM (2007) } \\
\text { AFV: } 20 \text { min } 3 \text { días a la } \\
\text { semana }^{\mathrm{R} 1}\end{array}$} & \multicolumn{4}{|c|}{$\begin{array}{c}\text { R2: PAG (2008) y WHO (2010) } \\
\text { AFV: } 75 \text { min semanales }{ }^{\mathrm{R} 2}\end{array}$} \\
\hline & \multicolumn{2}{|c|}{ Uniaxial } & \multicolumn{2}{|c|}{ Triaxial } & \multicolumn{2}{|c|}{ Uniaxial } & \multicolumn{2}{|c|}{ Triaxial } & \multicolumn{2}{|c|}{ Uniaxial } & \multicolumn{2}{|c|}{ Triaxial } & \multicolumn{2}{|c|}{ Uniaxial } & \multicolumn{2}{|c|}{ Triaxial } \\
\hline & No & $\chi^{2}$ & No & $\chi^{2}$ & No & $\chi^{2}$ & No & $\chi^{2}$ & No & $\chi^{2}$ & No & $\chi^{2}$ & $\mathrm{No}$ & $x^{2}$ & No & $\chi^{2}$ \\
\hline $\begin{array}{l}\text { Chicos } \\
\mathrm{N}=33\end{array}$ & $\begin{array}{l}22 \\
66 \%\end{array}$ & $\begin{array}{l}, 011 \\
p>, 05\end{array}$ & $\begin{array}{l}30 \\
90 \% \\
\end{array}$ & $\begin{array}{l}, 009 \\
p>, 05\end{array}$ & $\begin{array}{l}31 \\
93 \% \\
\end{array}$ & $\begin{array}{l}, 640 \\
p>, 05\end{array}$ & $\begin{array}{l}33 \\
100 \% \\
\end{array}$ & $\begin{array}{l}, 538 \\
p>, 05\end{array}$ & $\begin{array}{l}4 \\
12 \% \\
\end{array}$ & $\begin{array}{l}7,845 \\
\mathrm{p}<, 05\end{array}$ & $\begin{array}{l}10 \\
30 \% \\
\end{array}$ & $\begin{array}{l}9,751 \\
p<, 05\end{array}$ & $\begin{array}{l}10 \\
30 \% \\
\end{array}$ & $\begin{array}{l}14,308 \\
p<0,001\end{array}$ & $\begin{array}{l}19 \\
57 \% \\
\end{array}$ & $\begin{array}{l}23,211 \\
\mathrm{p}<, 001\end{array}$ \\
\hline $\begin{array}{l}\text { Chicas } \\
\mathrm{N}=62\end{array}$ & $\begin{array}{l}42 \\
67 \% \\
\end{array}$ & & $\begin{array}{ll}56 \\
90 \% \\
\end{array}$ & & $\begin{array}{l}59 \\
95 \% \\
\end{array}$ & & $\begin{array}{l}61 \\
98 \% \\
\end{array}$ & & 0 & & $\begin{array}{l}4 \\
6 \% \\
\end{array}$ & & $\begin{array}{l}2 \\
3 \% \\
\end{array}$ & & $\begin{array}{ll}7 \\
11 \% \\
\end{array}$ & \\
\hline $\begin{array}{l}\text { Total } \\
\mathrm{N}=95\end{array}$ & $\begin{array}{l}64 \\
67 \%\end{array}$ & & $\begin{array}{l}86 \\
90 \% \\
\end{array}$ & & $\begin{array}{l}90 \\
94 \%\end{array}$ & & $\begin{array}{l}94 \\
98 \%\end{array}$ & & $\begin{array}{l}4 \\
4 \%\end{array}$ & & $\begin{array}{l}14 \\
14 \%\end{array}$ & & $\begin{array}{l}12 \\
12 \%\end{array}$ & & $\begin{array}{l}26 \\
27 \%\end{array}$ & \\
\hline
\end{tabular}

Nota: R1: AFM un mínimo de 30 minutos, 5 días a la semana y AFV un mínimo de 20 minutos 3 días a la semana. R2: AFM/semana un mínimo 150 minutos y AFV/ semana 75 minutos en total.

\section{Discusión}

La etapa universitaria es un período crítico donde se produce un fuerte descenso en la práctica de AF. Ante los escasos estudios realizados en la población universitaria espańola con medición objetiva que analicen el grado de cumplimiento de las diferentes recomendaciones internacionales, y la falta de estudios que analicen los NAF utilizando diferentes puntos de corte, los resultados de nuestro estudio revelan que: a) existen diferencias en los NAF, según género a favor de los hombres, e independientemente de los puntos de corte utilizados, tanto en la AFM como en la AFV; b) encontramos diferencias en los minutos de AFM y AFV, según los puntos de corte utilizados; c) un gran porcentaje de la muestra estudiada cumple con las recomendaciones de AFM independientemente de los puntos de corte utilizados. Mientras si consideramos la AFV, el grado de cumplimiento solo está por encima de las recomendaciones en el caso de los hombres, y cuando utilizamos los puntos de corte para datos triaxiales; d) existe un mayor grado de cumplimiento de las recomendaciones de AF, cuando se aplican los puntos de corte para datos triaxiales y cuando se utiliza la R2.

La primera hipótesis de este estudio planteaba que los niveles de actividad física moderada y/o vigorosa (AFMV, medida en minutos/semana), variarían según los puntos de corte utilizados (uniaxiales y triaxiales) y según el género, siendo mayor en hombres que en mujeres.

La mayor AFM y la AFV obtenida cuando utilizamos los puntos de corte triaxiales en ambos géneros, indica que los resultados están influenciados por los puntos de corte utilizados a la hora de interpretar los datos recogidos, tal y como se ha manifestado en otros estudios previos (Mota, Valente, Aires, Silva, Santos, et al., 2007). Que los NAF registrados cuando utilizamos puntos de corte triaxiales sean más altos, puede explicarse porque su mayor precisión al registrar los movimientos en tres ejes, tanto en jóvenes como en adultos
(Eston, Rowlands \& Ingledew, 1998; Plasqui, Joosen, Kester, Goris \& Westerterp, 2005), mientras que si lo hacemos utilizando puntos de corte para datos uniaxiales mediremos en un solo eje, y por tanto podríamos infravalorar algunos movimientos, sobre todo considerando que el movimiento humano es multidimensional (Westerterp, 1999).

La elección de los puntos de corte es fundamental, ya que realizando la misma actividad las variaciones entre el cumplimiento de unas mismas recomendaciones puede variar enormemente. En el estudio desarrollado por Watson et al. (2014) se realizó una comparación entre nueve diferentes puntos de corte, seis de ellos generados a partir de actividades de laboratorio y tres de ellos generados con actividades diarias. Encontraron resultados de cumplimiento de recomendaciones que oscilaban entre el 6,3\% y el $98 \%$, dependiendo de los puntos de corte utilizados a pesar de que los participantes habían realizado las mismas actividades, encontrando menores porcentajes de cumplimiento en aquellos generados en laboratorio. La utilización de múltiples puntos de corte para adultos, dificultará la comparación de resultados entre diferentes investigaciones. Por otro lado, en el estudio donde se proponen los puntos de corte triaxiales utilizados en nuestro trabajo (Sasaki, et al., 2011), se señala una limitación que hay que tener en cuenta para interpretar nuestros datos: no se han tenido en cuenta actividades de la vida cotidiana para generar los límites de counts, entre las diferentes intensidades de AF, lo que puede hacer que actividades que serían de intensidad ligera cuando se utilizan puntos de corte uniaxiales, puedan ser clasificadas como moderadas, al no haber sido testadas utilizando los puntos de corte triaxiales.

Por otro lado, en la literatura científica hemos encontrado numerosos estudios que comparan dispositivos (e.g., GT1M uniaxiales versus GT3X triaxiales). Mientras algunos estudios señalan que se pueden utilizar los dos tipos de manera igualitaria (e.g., Robusto \& Trost, 2012), otros encuentran diferencias en relación a la medición de los counts en las ac- 
tividades realizadas, principalmente en las actividades ligeras, (e.g., Hänggi, Phillips \& Rowlands, 2013), así como Sasaki et al. (2011) que registraron diferencias entre los dispositivos con firmwares diferentes. Esto indica que no existe un consenso a la hora de comparar dispositivos uniaxiales y triaxiales, apuntando que no se pueden utilizar de manera similar. En este estudio, la comparativa que se realiza es en el análisis de los puntos de corte que están estipulados para clasificar los counts obtenidos por el mismo dispositivo, por lo que limitaciones que apuntan los estudios mencionados no aparecen. Sin embargo, no se han encontrado estudios que analicen los niveles de AF en base a puntos de corte uniaxiales y triaxiales, por lo que no se pueden establecer comparaciones.

En relación a la segunda parte de la hipótesis vinculada a las diferencias por género en los NAF, mientras que algunos estudios en población universitaria han mostrado que los niveles en mujeres son menores que en hombres (Hacıhasanoğlu, Yildırım, Karakurt, \& Sağlam, 2011; Troiano, et al., 2008), otros estudios no encuentran diferencias significativas (Behrens \& Dinger, 2003; Hagstromer, et al., 2010), como así también se constata en el estudio realizado en población universitaria espańola realizado con medidas objetivas (AriasPalencia, et al., 2015). Quizá el motivo, como señala AriasPalencia et al. (2015), es que los estudiantes universitarios tienen un calendario académico estable y esto podría explicar que los patrones de AF, durante este periodo, sean comunes en ambos géneros. Nuestros resultados constatan mayores NAF en hombres que en mujeres, pero concretamente solo en las AFV. Estos resultados pueden explicarse porque los varones suelen realizar más actividades organizadas de tipo deportivo que conllevan una mayor intensidad de práctica, mientras que las mujeres tienden a realizar actividades de más baja intensidad (Laguna, Lara \& Aznar, 2011; Vilhjalmsson \& Kristjansdottir, 2003). Es importante considerar que en muy pocos estudios en población adulta, aparecen por separado la AFM y la AFV (Colley, et al., 2011).

La segunda hipótesis hacía referencia a que un bajo porcentaje de participantes cumpliría las recomendaciones de prácticas de $\mathrm{AF}$, variando según la recomendación de práctica utilizada y encontrando diferencias significativas a favor del género masculino.

Nuestros resultados sugieren que un alto porcentaje, cumple con la R1 y R2 de AFM, tanto si utilizamos los puntos de corte para datos uniaxiales como triaxiales. Este porcentaje desciende sustancialmente, cuando consideramos las recomendaciones relativas a la $\mathrm{AFV}$, con ambos puntos de corte. Este bajo cumplimiento de las recomendaciones de AFV tanto en mujeres como en hombres (chicos: R1 12\% uniaxial y $30 \%$ triaxial; R2 30\% uniaxial y $57 \%$ triaxial y chicas: R1 $0 \%$ uniaxial y $6 \%$ triaxial R2 3\% uniaxial y $11 \%$ triaxial) es un aspecto preocupante, ya que el realizar de manera regular AFV ha mostrado mejores beneficios a nivel metabólico y físico (Hu, Jousilahti, Antikalinen, Katzmarzyk \& Touomilenhto, 2010; Lakka \& Laaksonen, 2007). Por tanto, se hace necesario implementar programas de intervención para mejorar de manera específica el cumplimiento de recomendaciones de AFV, sobre todo en relación a la $\mathrm{R} 1$ en la que los porcentajes son más bajos, ya que dicha recomendación implica el cumplirla por lo menos, tres días a la semana, un mínimo de 20 minutos diarios.

Estos datos son similares a los encontrados en un estudio que utilizó acelerómetros realizado en España con población universitaria (Arias-Palencia, et al., 2015). Por otro lado, en términos de AFMV, se han encontrado resultados que en los que la población universitaria española cumple en menor porcentaje las recomendaciones de AF medida con cuestionarios que el registrado en nuestro estudio: Práxedes et al. (2016) un 48.61\%, Romaguera et al. (2011) el 43\% y Varela-Mato et al. (2012) únicamente el 27\%. Estos datos son también refrendados fuera de la órbita de nuestro país (Hasse, et al., 2004; Irwin, 2004). Este alto grado de cumplimiento encontrado puede deberse, a que no hemos considerado a la hora de registrar la AF, fragmentos de $10 \mathrm{~min}$. de actividad (bouts). Tal como señalan algunos autores (Baptista, et al., 2012; Scheers, Philippaerts \& Lefevre, 2013), cuando los criterios utilizados para analizar el grado de cumplimiento son más flexibles, un mayor porcentaje de sujetos pueden cumplirlas. Es importante considerar que muchos estudios no separan la AFM de la AFV para analizar el grado de cumplimiento de las recomendaciones de AF, lo que ha dificultado las comparaciones entre nuestros resultados y otros estudios. Pensamos que es importante analizar estas dos intensidades por separado, ya que los diferentes organismos internacionales recomiendan, para los adultos, diferentes minutos/día para cada una de ellas. Además, en un reciente meta análisis (Plotnikoff, Costigan, Williams, Hutchesson, Kennedy, Robards, et al., 2015) señalan que de 28 estudios analizados, 18 consiguieron mejorar la AFM, los días de participación en AF y el número de METS pero no así en AFV de manera específica. Por lo tanto, es fundamental analizar ambas intensidades por separado tal y como marcan las recomendaciones internacionales de AF saludable (R1 y R2), lo que permitirá diseñar programas de intervención con estrategias concretas.

Por otro lado, tal y como aparece en nuestro estudio, el porcentaje de universitarios que cumple con las recomendaciones variará según la recomendación utilizada (AriasPalencia, et al., 2015). Esta controversia se pone también de manifiesto en el estudio realizado, en población adulta, por Thompson et al. (2009), en el que compararon 12 diferentes recomendaciones, llegando a la conclusión de que nueve de cada 10 hombres, podrían ser descritos como activos o insuficientemente activos, según la recomendación utilizada. Las recomendaciones que son aplicadas a población universitaria son las de población adulta, pero los estudiantes entre los 18 
y 23 años, franja habitual de edad en universitarios, tienen una serie de características particulares como por ejemplo: se encuentran en un periodo crítico de aumento de peso, ya que disminuye la práctica de AF por horarios exigentes en las titulaciones que cursan, y aumenta la ingesta calórica al salir en muchos casos de su entorno familiar (LaCaille, Dauner, Krambeer \& Pedersen, 2008). Estas características concretas de transición de la adolescencia a la adultez no se ven reflejadas en las recomendaciones. Hay un salto de los 60 minutos de AFMV diarias que tienen que cumplir los niños, niñas y adolescentes (WHO, 2010) a las R1 y R2 de adultos, cuyas edades pueden oscilar entre 18-64 años, con marcadas diferencias a nivel físico, psicológico y social (Dai, Wang $\&$ Morrison, 2014). Una propuesta que se puede extraer en base a esta investigación y de la literatura revisada es que los organismos e instituciones de salud internacionales deberían proponer unas recomendaciones de AF saludable específicas para la etapa universitaria, lo que facilitaría y aunaría la información y comparación de los NAF, así como el establecimiento de estrategias para aumentarla.

En relación con el estudio, hay una serie de limitaciones que tienen que ser nombradas. En primer lugar señalar que la muestra utilizada en el estudio es pequeńa, por lo que las conclusiones no pueden ser extrapoladas a la población universitaria en general. En segundo lugar, en relación con los acelerómetros, señalar que no registran de manera adecuada, actividades como la natación, ciclismo, patinaje, remo o levantar pesas, lo que puede provocar una infraestimación de la AF realizada. Por otro lado, no se ha tenido en cuenta el análisis de los bouts (bloques de $10 \mathrm{~min}$. seguidos de intensidad moderada o vigorosa) para el cumplimiento de las recomendaciones. Esto habría, por un lado, provocado un descenso en el cumplimiento de las mismas, pero, por el otro, no nos habría permitido separar la AFM de la AFV lo que se ajusta mejor a las recomendaciones internacionales de AF. En relación con la programación de los acelerómetros, señalar que existe una consideración a tener en cuenta para interpretar nuestros datos: el epoch time elegido es 10 segundos, agrupados posteriormente en el tratamiento de los datos en un minuto, tal y como se ha utilizado en numerosos estudios en población adulta (Matthews, et al., 2012). Este hecho puede explicar el menor número de minutos registrados de AFV, ya que epoch time más cortos, de un segundo por ejemplo, se han descrito como más precisos para detectarla (Nilsson, Ekelund, Yngve $\&$ Sjostrom, 2002).

Nuestro estudio muestra mayores NAF en los chicos tanto en la AFM como en la AFV, encontrando diferencias según los puntos de corte utilizados con mayores niveles para los triaxiales que los uniaxiales. Hay un bajo porcentaje de cumplimiento de las recomendaciones de AFV. Existe un mayor grado de cumplimiento cuando se utiliza la R2. Destacar que, en la literatura no se encuentran recomendaciones específicas para la población universitaria a pesar de que las recomendaciones establecidas para la población adulta abarcan una amplia franja de edad (de los 18 a los 64 años), existiendo grandes diferencias (Dai, et al., 2014). En la transición del instituto a la universidad se produce un descenso en la práctica de $\mathrm{AF}$, lo que pueden estar asociados con diferentes factores de tipo psicosocial como ambiental: el tipo de desplazamiento (activo o pasivo), las barreras psicosociales, la educación parental, etc. (e.g., Molina-García, Queralt, Castillo \& Sallis, 2015), por lo que medir los NAF de la forma más exacta posible es fundamental. Los estudiantes universitarios tienen características diferentes a los adultos más mayores, así como más oportunidades para participar en programas deportivos ofertados por las universidades. Por ello, sería conveniente establecer unas recomendaciones específicas y adaptadas a la población universitaria (situada entre los 18 y los 25 años), ya que puede presentar características muy diferentes a otros grupos de adultos. Teniendo en cuenta todo ello, se podrían diseñar programas de intervención más adaptados a esta población (Scheers, et al., 2013).

\section{Referencias}

1. Anderson, C. B., Hagströmer, M., \& Yngve, A. (2005). Validation of the PDPAR as an adolescent diary: effect of accelerometer cut points. Medicine and Science in Sports and Exercise, 37(7), 1224-1230.

2. Arias-Palencia, N. M., Solera-Martínez, M., Gracia-Marco, L., Silva, P., Martínez-Vizcaíno, V., Cañete-García-Prieto, J.,...\& SánchezLópez. (2015). Levels and Patterns of Objectively Assessed Physical Activity and Compliance with Different Public Health Guidelines in University Students. PloS one, 10(11), 1-15. doi:10.1371/journal. pone. 014197

3. Baptista, F.,Santos, D. A, Silva, A. M, Mota, J.,Santos, R., Vale, S., ... \& Sardinha, L. B. (2012). Prevalence of the Portuguese population attaining sufficient physical activity. Medicine and Science in Sports and Exercise, 44(3), 466-473.

4. Behrens, T. K., \& Dinger, M. K. (2003). A preliminary investigation of college students physical activity patterns. American Journal of Health Studies, 18(2), 169-172.
5. Cocca, A., Liukkonen, J., Mayorga, D., \& Viciana, J. (2014). Health related Physical Activity levels in Spanish youth and young adults. Perceptual and Motor Skills, 118(1), 247-260.

6. Cocca, A., Mayorga Vega, D., y Viciana Ramírez, J. (2013). Relación entre niveles de actividad física y placer de ser activos en estudiantes universitarios. Revista Iberoamericana De Psicología Del Ejercicio y El Deporte, 8(2), 359-372.

7. Colley, R. C., Garriguet, D., Janssen, I., Craig C.L., Clarke, J., \& Tremblay, M. S. (2011). Physical activity of Canadian adults: accelerometer results from the 2007 to 2009 Canadian Health Measures Survey. Health Reports, 1(22), 7-14.

8. Craig, C. L., Marshall, A. L., Sjöström, M., Ekelund, U., Yngve, A., Bauman, A. E., ... \& Oja, P. (2003). International physical activity questionnaire: 12-country reliability and validity. Medicine and Science in Sports and Exercise, 35(8), 1381-1395.

9. Dai, S., Wang, F., \& Morrison, H. (2014). Predictors of decreased PA 
level over time among adults: a longitudinal study. American journal of preventive medicine, 47(2), 123-130.

10. Downs, A., Van Hoomissen, J., Lafrenz, A., \& Julka, D. L. (2014). Accelerometer-measured versus self-reported physical activity in college students: Implications for research and practice. Journal of American College Health, 62(3), 204-212.

11. Eston, R. G., Rowlands, A.V., \& Ingledew, D. K. (1998). Validity of heart rate, pedometry, and accelerometry for predicting the energy cost of children's activities. Journal of applied physiology, 84(1), 362-371.

12. Freedson, P. S., Melanson, E., \& Sirard, J. (1998). Calibration of the Computer Science and Applications, Inc. accelerometer. Medicine and Science in Sports and Exercise, 30(5), 777-781.

13. Fritschi, J. O., Brown, W. J., \& Van Uffelen, J. G. (2014). On your feet: protocol for a randomized controlled trial to compare the effects of pole walking and regular walking on physical and psychosocial health in older adults. BMC Public Health, 14(1), 1-8.

14. Hacıhasanoğlu, R., Yıldırım, A., Karakurt, P., \& Sağlam, R. (2011) Healthy lifestyle behaviour in university students and influential factors in eastern Turkey. International Journal of Nursing Practice, 17(1), 43-51.

15. Hagstromer, M., Troiano, R. P., Sjostrom, M., \& Berrigan, D. (2010). Levels and patterns of objectively assessed physical activity - a comparison between Sweden and the United States. American Journal of Epidemiology, 171(10), 1055-1064.

16. Hänggi, J. M., Phillips, L. R. S., \& Rowlands, A. V. (2013). Validation of the GT3X ActiGraph in children and comparison with the GT1M ActiGraph. Journal of Science and Medicine in Sport, 16(1), 40-44.

17. Haskell, W. L., Lee, I. M., Pate, R. R., Powell, K. E., Blair, S. N., Franklin, B. A., ... \& Bauman, A. (2007). Physical activity and public health: updated recommendation for adults from the American College of Sports Medicine and the American Heart Association. Circulation 116(9), 1081-1093.

18. Hasse, A., Steptoe, A., Phil, D., Sallis, J. F., \& Wardle, J. (2004). Leisure-time physical activity in university students from 23 countries: associations with health beliefs, risk awareness, and national economic development. Preventive Medicine, 39(1), 182-190.

19. Hu, G., Jousilahti, P., Antikainen, R., Katzmarzyk, P.T \& Tuomilehto, J. (2010). Joint effects of physical activity, body mass index, waist circumference, and waist-to-hip ratio on the risk of heart failure. Circulation, 121(2), 237-244

20. Hultquist, C., Duckham, R., Stinson, C., \& Thompson, D. (2009). College Physical activity is related to mid-life activity levels in woman. Journal of Exercise Physiology online, 12(4), 1-7.

21. Irwin, J. D. (2004). Prevalence of university students' sufficient physical activity: A systematic review. Perceptual and Motor Skills, 98(3), 927-943.

22. La Caille, L.J, Dauner, K.N, Krambeer, R.J., \& Pedersen J. (2008). Psychosocial and environmental determinants of eating behaviors, physical activity, and weight change among college students: a qualitative analysis. Journal of American College Health, 59(6), 531-538

23. Laguna, M., Lara, M. T., \& Aznar, S. (2011). Patrones de Actividad Física en función del género y los niveles de obesidad en población infantil espańola. Estudio EYHS. Revista de Psicología del Deporte, 20(2), 621-631.

24. Lakka, T.A., \& Laaksonen, D.E. (2007). Physical activity in prevention and treatment of the metabolic syndrome. Applied physiology, nutrition and metabolism. 32(1), 76-88.

25. López Sánchez, G. F., Ahmed, D., \& Díaz Suárez, A. (2017). Level of habitual physical activity among 13-year-old adolescents from Spain and India. A cross-cultural study. SPORT TK: Revista EuroAmericana de Ciencias del Deporte, 6(1), 67-74

26. López Sánchez, G. F., González Víllora, S., \& Díaz Suárez, A. (2016). Level of habitual physical activity in children and adolescents from the Region of Murcia (Spain). SpringerPlus, 5:386, 1-6. http://dx.doi org/10.1186/s40064-016-2033-8

27. Matthews, C. E. (2005). Calibration of accelerometer output for adults. Medicine and Science in Sports and Exercise, 37(11), 512-522.

28. Matthews, C. E., Hagstromer, M., Pober, D. M., \& Bowles, H. R. (2012). Best practices for using physical activity monitors in population-based research. Medicine and Science in Sports and Exercise, 44(1), $68-76$.

29. Molina-García, J., Castillo, I., \& Pablos, C. (2009). Determinants of leisure-time physical activity and future intention to practice in Spanish college students. The Spanish Journal of Psychology, 12(1), 128-137

30. Molina-García, J., Queralt, A., Castillo, I., \& Sallis, J. F. (2015). Changes in Physical Activity domains during the transition out of high school: psychosocial and environmental correlates. Journal of Physical Activity and Health. doi:0.1123/jpah.2014-0412.

31. Mota, J.,Valente, M., Aires, L., Silva, P., Santos, M. P., \& Ribeiro, J. C. (2007). Accelerometer cut-points and youth physical activity prevalence. European Physical Education Review, 13(3), 287-299.

32. Nilsson, A., Ekelund, U., Yngve, A., \& Sjostrom, M. (2002). Assessing physical activity among children with accelerometers using different time sampling intervals and placements. Pediatric Exercise Science, 14(1), 87-96.

33. Oja, P., Bull, F. C., Fogelholm, M., \& Martin, B. W. (2010). Physical activity recommendations for health: what should Europe do? BMC Public Health, 10(1), 1-10. doi: 10.1186/1471-2458-10-10

34. Ortega, F. B., Konstabel, K., Pasquali, E., Ruiz, J. R., Hurtig-Wennlöf, A., Mäestu, J., ... \& Sjöström, M. (2013). Objectively measured physical activity and sedentary time during childhood, adolescence and young adulthood: a cohort study. PloS one, 8(4), e60871. doi:10.1371/ journal.pone.0060871

35. Pate, R. R., Pratt, M., Blair, S. N. , Haskell, W. L., Macera, C. A. Bouchard, C., ... \& Wilmore, J. H. (1995). Physical activity and public health: a recommendation from the Centers for Disease Control and Prevention and the American College of Sports Medicine. Jama, 273(5), 402-407.

36. Plasqui, G., Joosen, A. M., Kester, A. D., Goris, A. H., \& Westerterp, K. R. (2005). Measuring free-living energy expenditure and physical activity with triaxial accelerometry. Obesity Research, 13(8), 13631369.

37. Plotnikoff, R.C., Costigan, S.A., Williams, R.L., Hutchesson, M.J., Kennedy, S.G.,Robards, S.L.,...\& Germov,J . (2015).Effectiveness of interventions targeting physical activity, nutrition and healthy weight for university and college students: a systematic review and meta-analysis. International Journal of Behavioral Nutrition and Physical Activity, 12, 45-015-0203-7. doi:10.1186/s12966-015-0203-7

38. Práxedes, A., Sevil, J., Moreno, A., del Villar, F., \& García-González, L. (2016). Niveles de actividad física en estudiantes universitarios: diferencias en función del género, la edad y los estados de cambio. Revista Iberoamericana de Psicología del Ejercicio y el Deporte, 11(1), 123-132.

39. Prince, S., Adamo, K., Hamel, M., Hardt, J., Gorber, S., \& Tremblay, M. (2008). A comparison of direct versus self-report measures for assessing physical activity in adults: a systematic review. International Journal of Behavioral Nutrition and Physical Activity, 5(1), 56. doi:10.1186/14795868-5-56

40. Racette, S. B., Deusinger, S. S., Strube, M. J., Highstein, G. R., \& Deusinger, R. H. (2005). Weight changes, exercise, and dietary patterns during freshman and sophomore years of college. Journal of American College Health, 53(6), 245-251.

41. Romaguera, D., Tauler, P., Bennasar, M., Pericas, J., Moreno, C., Martínez, S., et al. (2011). Determinants and patterns of physical activity practice among Spanish university students. Journal of Sports Sciences, 29(9), 989-997.

42. Ridgers, N.D., \& Fairclough, S. (2011). Assessing free-living physical 
activity using accelerometry: Practical issues for researchers and practitioners. European Journal of Sport Science, 11, 205-213.

43. Robusto, K. M. \& Trost, S. G. (2012). Comparison of three generations of Actigraph $^{\mathrm{TM}}$ activity monitors in children and adolescents. Journal of Sports Science, 30(13), 1429-1435.

44. Sasaki, J. E., John, D., \& Freedson, P. S. (2011). Validation and comparison of ActiGraph activity monitors. Journal of Science and Medicine in Sport, 14(5), 411-416.

45. Scheers, T.; Philippaerts, R., \& Lefevre, J. (2013). Compliance with different physical activity recommendations and its association with socio-demographic characteristics using an objective measure. BMC Public Health, 13(1), 1.

46. Sparling, P. B., \& Snow, T. K. (2002). Physical activity patterns in recent college alumni. Research Quarterly for Exercise and Sport, 73(2), 200-205.

47. Thompson, D., Batterham, A. M., Markovitch, D., Dixon, N. C., Lund, A. J., \& Walhin, J. P. (2009). Confusion and conflict in assessing the physical activity status of middle-aged men. PLoS One, 4(2), e4337. doi:10.1371/journal.pone.0004337

48. Timperio, A., Salmon, J., \& Ball, K. (2004). Evidence-based strategies to promote physical activity among children, adolescents and young adults: review and update. Journal of Science and Medicine in Sport, 7(1), 20-29.

49. Troiano, R. P., Berrigan, D., Dodd, K. W. , Masse, L.C., Tilert, T., \& McDowell, M. (2008). Physical activity in the United States measured by accelerometer. Medicine and Science in Sports and Exercise, 40(1), 181-188.

50. Trost, S. G., McIver, K. L., \& Pate, R. R. (2005). Conducting accelero- meter-based activity assessments in field-based research. Medicine and Science in Sports and Exercise, 37(11), 531-543.

51. Varela-Mato, V., Cancela, J. M., Ayan, C., Martín, V., \& Molina, A. (2012). Lifestyle and health among Spanish university students: differences by gender and academic discipline. International Journal of Environmental Research and Public Health, 9(8), 2728-2741.

52. Vilhjalmsson, R., \& Kristjansdottir, G. (2003). Gender differences in physical activity in older children and adolescents: the central role of organized sport. Social Science and Medicine, 56(2), 363-374.

53. Vin̄as, B. R., Barba, L. R., Ngo, J., \& Majem, L. S. (2013). Validación en población catalana del cuestionario internacional de actividad física. Gaceta Sanitaria, 27(3), 254-257.

54. Watson, K. B., Carlson, S., Carrol, D. D., \& Fulton, J. (2014). Comparison of Accelerometer Cut Points to Estimate Physical Activity in U.S. Adults. Journal of Sports Science, 32(7) 660-669.

55. Welk, G. J. (2002). Use of accelerometry-based activity monitors to assess physical activity. Physical activity assessments for health-related research, 125-141.

56. Westerterp, K. R. (1999). Assessment of physical activity level in relation to obesity: current evidence and research issues. Medicine and Science in Sports and Exercise, 31(11), 522-525.

57. World Health Organization. (2010). Global Recommendations on Physical Activity for Health. WHO Press: Geneva, Switzerland.

58. Yıldırım, M., Verloigne, M., De Bourdeaudhuij, I., Androutsos, O., Manios, Y., Felső, R., ... \& Chinapaw, M. J. M. (2011). Study protocol of physical activity and sedentary behaviour measurement among schoolchildren by accelerometry-Cross-sectional survey as part of the ENERGY-project. BMC public health, 11(1). 